\title{
Hyaluronic acid reagent functional chitosan-PEI conjugate with AQP2-siRNA suppressed endometriotic lesion formation
}

This article was published in the following Dove Press journal:

International Journal of Nanomedicine

30 March 2016

Number of times this article has been viewed

\author{
Meng-Dan Zhao' \\ Jin-Lin Cheng ${ }^{2}$ \\ Jing-Jing Yan' \\ Feng-Ying Chen' \\ Jian-Zhong Sheng ${ }^{3}$ \\ Dong-Li Sun' \\ Jian Chen ${ }^{4}$ \\ Jing Miao ${ }^{4}$ \\ Run-Ju Zhang' \\ Cai-Hong Zheng' \\ He-Feng Huang ${ }^{1,5}$ \\ 'Women's Hospital, School of \\ Medicine, Zhejiang University, \\ Hangzhou, ${ }^{2}$ State Key Laboratory \\ for Diagnosis and Treatment of \\ Infectious Diseases, Collaborative \\ Innovation Center for Diagnosis and \\ Treatment of Infectious Diseases, \\ The First Affiliated Hospital, College \\ of Medicine, ${ }^{3}$ Department of \\ Pathophysiology, School of Medicine, \\ ${ }^{4}$ The First Affiliated Hospital, College \\ of Medicine, Zhejiang University, \\ Hangzhou, ${ }^{5}$ International Peace \\ Maternity and Child Health Hospital, \\ School of Medicine, Shanghai Jiao \\ Tong University, Shanghai, People's \\ Republic of China
}

Correspondence: He-Feng Huang; Cai-Hong Zheng

Women's Hospital, School of Medicine, Zhejiang University, I Xueshi Road, Hangzhou 310006, People's Republic of China

Email hhf@zju.edu.cn; chzheng@zju.edu.cn
Abstract: To identify a new drug candidate for treating endometriosis which has fewer side effects, a new polymeric nanoparticle gene delivery system consisting of polyethyleniminegrafted chitosan oligosaccharide (CSO-PEI) with hyaluronic acid (HA) and small interfering RNA (siRNA) was designed. There was no obvious difference in sizes observed between (CSO-PEI/siRNA)HA and CSO-PEI/siRNA, but the fluorescence accumulation in the endometriotic lesion was more significant for (CSO-PEI/siRNA)HA compared with CSO-PEI/siRNA due to the specific binding of HA to CD44. In addition, the (CSO-PEI/siRNA)HA nanoparticle gene therapy significantly decreased the endometriotic lesion sizes with atrophy and degeneration of the ectopic endometrium. The epithelial cells of ectopic endometrium from rat models of endometriosis showed a significantly lower CD44 expression than control after treatment with (CSO-PEI/siRNA)HA. Furthermore, observation under an electron microscope showed no obvious toxic effect on the reproductive organs. Therefore, (CSO-PEI/siRNA)HA gene delivery system can be used as an effective method for the treatment of endometriosis.

Keywords: chitosan-PEI, hyaluronic acid, AQP2-siRNA, endometriosis, targeted therapy

\section{Introduction}

The definition of endometriosis is the appearance of official endometrial tissues outside the endometrium and the myometrium, thus affecting women of childbearing age, causing a decline in health, due to disorders such as dysmenorrhea, chronic pelvic pain, and infertility. The real mechanisms and pathophysiological basis of endometriosis are yet to be completely understood. ${ }^{1}$ The widely accepted etiology of the disease is the implantation of endometrial tissue in retrograde menstruation flux. ${ }^{2}$

The available clinical treatment is surgical removal of endometriotic tissues and uterus or drugs targeted at decreasing or arresting the production of cyclic ovarian hormones. However, drugs used in conservative treatment of endometriosis, for example, progesterone, gonadotropin-releasing hormone, danazol, angiogenesis disruption, and antitumor necrosis factor-alpha inhibitors, ${ }^{3,4}$ are often connected with numerous side effects, limiting their use for long-term treatment.

Therefore, studies for identifying a new drug candidate for treating endometriosis with fewer side effects are very important. Nucleic acid therapy is playing an increasingly important role in gene therapy and nucleic acid therapy has achieved great success in the currently intractable diseases. ${ }^{5}$ Aquaporins (AQPs) are a family of transmembrane proteins, widely distributed throughout various tissues, and play an important role by promoting response to changing osmotic gradient across the cell membrane transport, 
such as glycerol water, to maintain intra-/extracellular fluid homeostasis. ${ }^{6,7}$ Our previous studies have demonstrated that AQP2 is expressed in endometrial tissues from patients with endometriosis, which is an estrogen-dependent disease, and AQP2 knockdown attenuated estradiol-enhanced cell migration, invasion, and adhesion. ${ }^{8}$

Small interfering RNA (siRNA) is a powerful and highly specific tool for gene silencing, which has been widely used to study target discovery and gene function. ${ }^{9}$ In this study, we used the AQP2-siRNA to treat endometriosis. However, siRNA delivery is difficult to achieve the targeted tissue with high efficiency in vivo. In clinical treatment, the delivery system needs to protect the siRNA from degradation in order to enhance cellular uptake and transport to target tissue. In fact, it is quite difficult to effectively deliver genes for gene therapy with nonviral gene delivery system.

Recently, we have demonstrated highly efficient in vitro delivery of DNA in an orthotopic model of cancer by using novel chitosan oligosaccharide (CSO)-stearic acid micelles. ${ }^{10,11}$ In addition, we also demonstrated the feasibility of endometriosis treatment by using chitosan-derived micelles/pigment epithelium-derived factor gene delivery system. ${ }^{12}$ However, the efficiency of transfection is not satisfactory with this kind of gene delivery. For further study on gene therapy for endometriosis, we need to improve our nonviral gene delivery systems.

Polyethylenimine (PEI) is one of the most effective cationic polymers with a high charge density potential for nonviral gene delivery. Due to the proton sponge effect, PEI was provided with high transfection efficiency and high levels of transgenic expression in mammalian cell types. ${ }^{13}$ However, significant toxicity, such as that of commercial PEI, heterogeneous structure, and lack of options for precise chemical modification are the disadvantages of this polymer. ${ }^{14}$ It is generally considered that low-molecular-weight PEI shows low transfection efficiency with low cytotoxicity, which limits its use in gene delivery systems. ${ }^{15}$ Several studies conducted to overcome these limitations have proven that decorating lower molecular-weight PEI with an organic macromolecule resulted in lower cytotoxicity and satisfactory transfection efficiency. ${ }^{11}$ Chitosan found in crustacean shells with a natural polysaccharide has been widely used for gene delivery, as we have shown in previous studies. ${ }^{12}$ In the present study, lower molecular-weight PEI 800 was conjugated to $\mathrm{CSO}$ with different amino-substituted degrees to create novel polycationic gene carriers, and transfection of siRNA was mediated by nanoparticles to ameliorate the gene transfection efficiency. We also studied the biophysical characterization of the CSO-PEI/siRNA systems coated with hyaluronic acid (HA). HA, a polyanionic polysaccharide distributed widely in the extracellular matrix and the joint liquid of mammalians, has a low toxicity, biodegradable, and biocompatible and was approved by the US Food and Drug Administration for injections. ${ }^{16} \mathrm{HA}$ and its products induce receptor-mediated intracellular signaling, such as endocytosis, degradation, and signal transduction. ${ }^{17} \mathrm{CD} 44$, which was identified as HA receptor, was found to be overexpressed in endometriotic cells. ${ }^{18}$ Interestingly, there was no effect on binding characteristics of HA-treated endometrial cell to mesothelial cell, suggesting that endometrial cells might have a potential to bind to HA of surrounding tissue. ${ }^{2}$

So, it is a powerful method to shield the positive charge of gene vectors with the targeting ligands (HA) in the guidance of the concept of receptor-mediated endocytosis, which was aimed at directing gene delivery to desired tissues. The endometriosistargeting moiety of a delivery vehicle plays an important role in efficient treatment by not only reducing unwanted systemic toxicity but also overcoming dose limitation. ${ }^{19}$ Therefore, we selected PEI and CSO systems, which were previously developed by our research group, and modified them by using HA to increase the localization of the polyplex in endometriosis microenvironment (Figure 1A). Thus, we synthesized a novel gene carrier (CSO-PEI/siRNA)HA and evaluated its physicochemical properties, in vitro cytotoxicity and transfection efficiency, cellular uptake efficiency, in vivo biodistribution, therapeutic effect, and initial mechanism of siRNA gene therapy and explored the feasibility of siRNA gene therapy on endometriosis.

\section{Materials and methods Materials}

$\mathrm{CSO}(\mathrm{Mw}=17.5 \mathrm{kDa}, 95 \%$ deacetylated degree$)$ was obtained by enzymatic degradation of chitosan $(\mathrm{Mw}=450.0 \mathrm{kDa})$. PEI $(\mathrm{Mw}=800 \mathrm{Da})$ and ethidium bromide was purchased from Sigma Chemicals, Perth, Australia. 1-Ethyl-3-(3dimethylaminopropyl) carbodiimide was purchased from Shanghai Medpep Co., Ltd., Shanghai, People's Republic of China. 2,4,6-Trinitrobenzene sulfonic acid and 3-(4,5dimethylthiazol-2-yl)-2,5-diphenyltetrazolium bromide (MTT) were purchased from Sigma Chemicals. Dimethyl sulfoxide was purchased from Haishuo Biochemistry Co., Ltd., Wuxi, People's Republic of China. Trypsin, DNase I, and Dulbecco's Modified Eagle's Medium were purchased from Thermo Fisher Scientific, Waltham, MA, USA. Fetal bovine serum (FBS) was purchased from Sijiqing Biological Engineering Materials Co. Ltd., Hangzhou, People's Republic of China. AQP2 siRNA duplexes were chemically synthesized by Ambion (Carlsbad, CA, USA). The integrity 


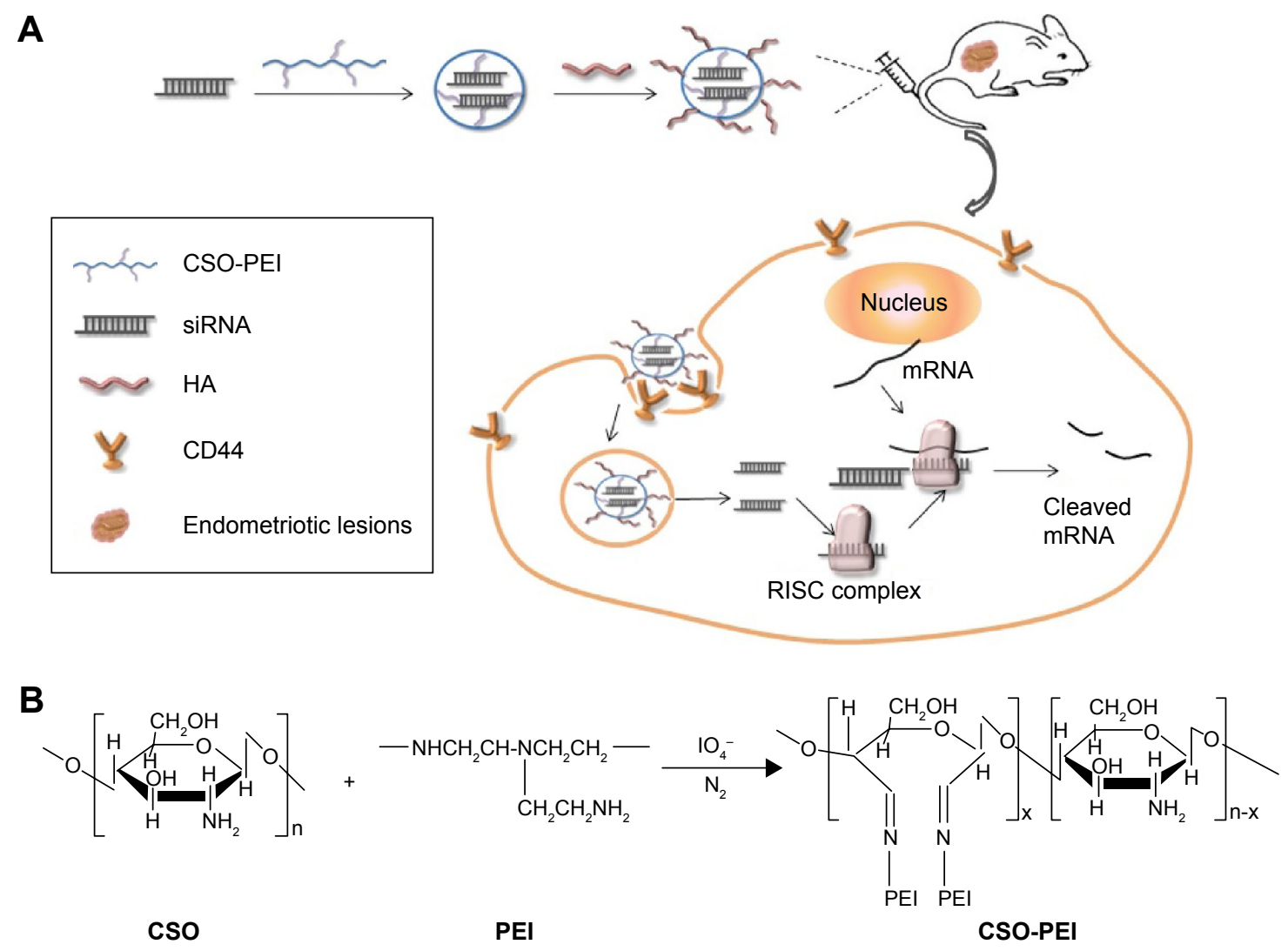

Figure I (A) The polyplex in endometriosis microenvironment; (B) synthesis route for the CSO-PEl chemical conjugate.

Abbreviations: CSO-PEI, polyethylenimine-grafted chitosan oligosaccharide; siRNA, small interfering RNA; HA, hyaluronic acid; RISc complex, RNA-induced silencing complex.

of plasmids was identified by agarose gel electrophoresis. Its purity and concentration were determined by ultraviolet absorbance at 260 and $280 \mathrm{~nm}$. All other solvents were of analytical grade.

Trichloroacetaldehyde hydrate was purchased from Sinopharm Chemical Reagent Co., Ltd. (Shanghai, People's Republic of China); physiologic saline from Hangzhou Minsheng Pharmaceutical Co., Ltd. (Zhejiang, People's Republic of China); and penicillin from North China Pharmaceutical Co., Ltd. (Shijiazhuang, People's Republic of China). Mouse monoclonal antibodies against von Willebrand factor (vWF, M0616) and $\alpha$-smooth muscle actin ( $\alpha$-SMA, M0851) were applied by Dako Denmark A/S (Glostrup, Denmark). Diaminobenzidine (DAB) (GK346810) and EnVision+/ horseradish peroxidase/mouse (GK400105) was purchased from Novocastra Laboratories Ltd. (Newcastle Upon Tyne, UK). The In Situ Cell Death Detection Kit, fluorescein (11684817910) was provided by Hoffman-La Roche Ltd. (Basel, Switzerland). Danazol was from Yangzhou Pharmaceutical Co., Ltd. (Jiangsu, People's Republic of China). Ethanol and other chemicals were of analytical grade and used without further purification.

\section{Cell culture}

Ishikawa (IK) cells (American Type Culture Collection, Manassas, VA, USA), a human endometrial adenocarcinoma cell line, were cultured in phenol red Roswell Park Memorial Institute-1640 medium supplemented with 10\% FBS and $1 \%$ penicillin at $37^{\circ} \mathrm{C}$ and a humidified atmosphere containing $5 \% \mathrm{CO}_{2}$.

\section{Surgical induction of endometriosis rat model}

Six-week-old nonpregnant female Sprague Dawley rats weighing 200-250 g were purchased from Shanghai Animal Center, Chinese Academy of Science (Shanghai, People's Republic of China) and housed in the Key Laboratory of Combined Multi-Organ Transplantation, Chinese Ministry of Public Health, First Affiliated Hospital, School of Medicine, Zhejiang University. Rats were fed with sterilized water and standard rat chow under a controlled environment. All animals were maintained in accordance with the National Institutes of Health Guide for the Care and Use of Laboratory Animals, and the experiment was approved by the Experimental Animal Ethics Committee of Zhejiang University. 
All rats were acclimated for at least 1 week before the operation, and only rats exhibiting regular 4-5-day estrous cycles were used.

Endometriosis lesion animal model was created by transplanting autologous fragments of uterus to the inner surface of the abdominal wall, as reported by Vernon and Wilson. ${ }^{20}$

\section{Synthesis of CSO-PEI}

CSO-PEI copolymer was synthesized in two steps according to a previous report, with some modification. ${ }^{11} \mathrm{CSO}(0.25 \mathrm{~g})$ and sodium periodate $(0.01 \mathrm{~g})$ were each dissolved in sodium acetate buffer ( $\mathrm{pH} 4.5$ ) degassed with $\mathrm{N}_{2}$. After mixing the solutions, the reaction mixture was stirred for 2 days at $4^{\circ} \mathrm{C}$. Then, the reaction was stopped by adding ethylene glycol. The reaction solution was dialyzed against deionized water for 8 hours with frequent exchange of fresh deionized water. Then, PEI solution was added dropwise to periodate-oxidized $\mathrm{CSO}$ solution. The reaction was carried out for 48 hours at $4^{\circ} \mathrm{C}$ under stirring at $300 \mathrm{rpm}$. At the end of the reaction, the solution was treated with sodium borohydride. Finally, after dialyzing against deionized water for 24 hours, the dialyzed product was lyophilized, and the CSO-PEI was synthesized.

\section{Preparation and characterization of (CSO-PEI/siRNA)HA complex nanoparticle}

The solutions of CSO-PEI of different concentrations were coated by HA and then purified by $0.22 \mu \mathrm{m}$ Millipore filter (Millipore, Massachusetts, USA). The stable (CSO-PEI/ siRNA)HA complex nanoparticles with various N/P ratios were prepared by mixing the appropriate volume of (CSOPEI)HA and siRNA solution vortically for 30 seconds and then letting it stand for 30 minutes.

The average hydrodynamic diameter and zeta potential of (CSO-PEI/siRNA)HA complex nanoparticles were measured by dynamic light scattering using a Zetasizer (3000HS; Malvern Instruments, Malvern, UK). The morphological examination of (CSO-PEI/siRNA)HA-complex nanoparticle was performed by atomic force microscopy (AFM) (SPA 3800N; Seiko, Tokyo, Japan) using tapping mode and high resonance frequency pyramidal cantilevers with silicon probes having force constants of $20 \mathrm{~N} / \mathrm{m}$. Scan speeds were set at $2 \mathrm{~Hz}$.

\section{Agarose gel electrophoresis experiments}

Varying amounts of (CSO-PEI)HA and siRNA were combined in different weight ratios and mixed with gentle vortex. The mixtures were incubated at room temperature for 30 minutes prior to loading. Electrophoresis was then carried out with a current of $120 \mathrm{~V}$ for 30 minutes in tris, acetate, and EDTA buffer solution. siRNA retardation was visualized by staining with ethidium bromide and exposure to ultraviolet light.

\section{In vitro cytotoxicity assay}

Cytotoxicity of (CSO-PEI)HA against IK cells was evaluated by MTT assay. Briefly, $10^{4}$ cells were seeded in a 96 -well plate with $0.2 \mathrm{~mL}$ of growth medium containing $10 \% \mathrm{FBS}$ and antibiotics. Cells were cultured at $37^{\circ} \mathrm{C}$ for 24 hours prior to the addition of filtered test materials. Then, the growth medium was replaced by fresh medium containing the desired amount of test materials. The cells were further incubated for 48 hours. At the end of incubation, $15 \mu \mathrm{L}$ of MTT solution was added to each well, and cells were incubated for additional 4 hours. After that, the medium was removed and $200 \mu \mathrm{L}$ of dimethyl sulfoxide was then added to each well to dissolve the MTT formazan crystals. Plates were shaken for 15 minutes, and the absorbance was read on a microplate reader (Model 680; BioRad Laboratories Inc., Hercules, CA, USA) at a wavelength of $570 \mathrm{~nm}$.

\section{Cellular uptake}

For investigating the intracellular distribution in cells, (CSOPEI)HA was further labeled with fluorescein isothiocyanate (FITC) via the reactive amino group and the isothiocyanate group of FITC.

The IK cells were seeded for 24 hours in 24-well plates in $1 \mathrm{~mL}$ of 1640 medium. Before uptake experiments, the medium in each well was replaced with $1 \mathrm{~mL}$ of fresh culture medium. The cells were incubated for 1, 2, and 8 hours with FITC(CSO-PEI)HA or FITC-(CSO-PEI) and were washed three times with ice-cold phosphate buffers ( $\mathrm{pH} 7.2)$ to terminate the uptake. Nuclei were stained using 4',6-diamidino-2phenylindole. The cells were observed under fluorescence microscopy (Leica Microsystems, Wetzlar, Germany).

\section{Distribution of (CSO-PEl/siRNA)HA in animal model}

The DiR was used as a fluorescent probe and was loaded into (CSO-PEI/siRNA)HA for in vivo fluorescent imaging investigation. After tail vein injection of (CSO-PEI/siRNA) HA/DiR, the mice were anesthetized by diethyl ether and were observed by the Maestro in vivo imaging system (Cambridge Research \& Instrumentation Inc., Woburn, MA, USA) at the predetermined time. For ex vivo analysis, the mice were sacrificed, and in vitro tissues together with cysts were collected and weighed. The fluorescent intensity, reflecting the amount of nanoparticles, was also read by the imaging system. The accumulation of (CSO-PEI/siRNA)HA/DiR 
in various tissues was expressed as the percentage of the injected dose per gram of tissue $(\% \mathrm{ID} / \mathrm{g})$.

\section{Animal treatment}

Rats were divided into three different groups consisting of nine rats each. The first group, negative control, was given $1.5 \mathrm{~mL}$ of sterile normal saline via intravenous (IV) injection. The second group, positive control, was given $36 \mathrm{mg} / \mathrm{kg}$ body weight of oral danazol daily (200 mg) for 2 weeks via gastric gavage. The third group was treated with (CSO-PEI/ siRNA)HA and was injected with $5 \mathrm{mg} / \mathrm{kg}$ body weight of siRNA.

The size of the cyst and the body weight of each rat were measured every week thereafter. After 2 weeks, the rats were sacrificed and each cyst was excised and measured. At the same time, endometriotic lesions in control and drugtreated groups were observed under light microscopy (Leica Microsystems).

The inhibition of cyst growth was calculated using the following formula: $\mathrm{IR}$ (inhibition rate) $(\%)=(\mathrm{Wc}-\mathrm{Wt}) / \mathrm{Wc} \times 100 \%$; where $\mathrm{Wc}$ is the average cyst size of controlled group and $\mathrm{Wt}$ is the average cyst size of treated group.

Meanwhile the intact uterus and ovary of the rats were weighed to calculate the visceral index and examined under light microscopy.

\section{Transmission electron microscopy for observation of reproductive organs}

Transmission electron microscopy observation was carried out to find whether (CSO-PEI/siRNA)HA caused changes in the structure of the uterus and ovary, especially apoptosis of the cells.

A part of the uteri and ovaries were removed and fixed in $2.5 \%$ glutaraldehyde overnight in a refrigerator and washed three times with phosphate-buffered saline for 15 minutes. Then, it was placed in $1 \%$ osmium tetroxide for 1 hour and washed three times with phosphate-buffered saline. After that, the samples were stained in $4 \%$ uranyl acetate for 30 minutes, then dehydrated by series concentration of ethanol and embedded in resin mixture. This was sectioned and observed under a TECNAI-10 transmission electron microscope (FEI Company, Hillsboro, OR, USA).

\section{Immunohistochemical analysis}

To explore the vascular response to (CSO-PEI/siRNA)HA, the microvessel density (MVD) of the endometrial implants was evaluated. Monoclonal mouse anti-vWF and monoclonal mouse anti- $\alpha$-SMA were used to determine the MVD of endometrial lesions.
Formalin-fixed, paraffin-embedded tissues were cut into $4 \mu \mathrm{m}$ thick sections. These sections were deparaffinized and rehydrated in a graded series of ethanol following standard protocol and then treated with $3 \% \mathrm{H}_{2} \mathrm{O}_{2}$ for 10 minutes to eliminate endogenous peroxidase activity. Sections were immunostained using the monoclonal mouse anti-vWF antibody and $\alpha$-SMA antibody for 60 minutes at room temperature. Sections were washed with phosphate-buffered saline and incubated with EnVision-labeled polymer-alkaline phosphates mouse (EnVision+/horseradish peroxidase/mouse, GK400105; Novocastra Laboratories Ltd.) for 60 minutes. The antigen-antibody reaction was visualized using $\mathrm{DAB}$ as chromogen (GK346810; Novocastra Laboratories Ltd.). After washing with phosphate-buffered saline, the sections were counterstained with hematoxylin, then dehydrated, and mounted on a mounting medium.

The MVD was determined from the number of VWF- and $\alpha$-SMA-positive vessels, indicated by the presence of cytoplasmic brown staining. Any distinct single brown-stained cell or cluster of endothelial cells was considered to be a microvessel with or without lumen formation or red blood cells. MVD was calculated as described previously. ${ }^{21}$

\section{CD44 detection}

Formalin-fixed and paraffin-embedded tissues were cut into $4 \mathrm{~mm}$ thick sections. These sections were deparaffinized and rehydrated in a graded series of ethanol following a standard protocol, subjected to antigen retrieval for 3 minutes with citrate buffer in a high pressure steamer, naturally cooled to room temperature, and then treated with $3 \% \mathrm{H}_{2} \mathrm{O}_{2}$ for 10 minutes to eliminate endogenous peroxidase activity. Sections were immunostained using the monoclonal rabbit anti-CD44 antibody for 30 minutes in an incubator at $37^{\circ} \mathrm{C}$. The antigen-antibody reaction was visualized using DAB as chromogen (GK346810; Novocastra Laboratories Ltd.).

\section{Statistical analysis}

Data were expressed as means of three or more separate experiments, and statistical analysis was performed using the Student's $t$-test. $P<0.05\left(^{*}\right)$ was considered to be significant in all cases.

\section{Results}

\section{Preparation and characterization of nanoparticle complexes}

CSO with the average molecular weight of $\sim 17.5 \mathrm{kDa}$ was synthesized by enzymatic degradation of $95 \%$ deacetylated chitosan $(\mathrm{Mw}=450.0 \mathrm{kDa})$. A multistep reaction for the synthesis of CSO-PEI vector construct was performed by 
combining PEI covalently linked to a CSO backbone. The route of synthesis is shown in Figure 1B. CSO-PEI conjugate was synthesized by imine reaction between amine group of PEI and aldehyde group of periodate-oxidized CSO (Figure 1B).

The (CSO-PEI)HA can efficiently compact siRNA into small and stable complexes with increasing cellular uptake and release of siRNA. To prepare nanosize siRNA polyplexes, the siRNA was compounded with the CSO-PEI and (CSO-PEI)HA. The hydrodynamic diameters of (CSO-PEI/ siRNA)HA polyplexes were measured at different N/P ratios from 0.1 to 16 (Figure 2 ). At the $\mathrm{N} / \mathrm{P}$ ratio $>2$, the (CSO-PEI)HA effectively condensed siRNA into small polyplexes with a diameter of $<200 \mathrm{~nm}$. The siRNA condensation capacity with (CSO-PEI)HA was also studied at various N/P ratios of (CSO-PEI)/siRNA by gel retardation assay. Figure $2 \mathrm{~A}$ and $\mathrm{B}$ shows the gel retardation results of the complexes with N/P ratio from 0.1 to 16 . When the N/P ratio was $>2$, the complete retardation of the complexes could be seen, suggesting that (CSO-PEI)HA and siRNA started to form tight complexes.

Figure $3 \mathrm{~A}$ shows the size distribution of (CSO-PEI/siRNA) HA and CSO-PEI/siRNA (N/P =4) complexes, respectively. The average volume diameter of CSO-PEI/siRNA with $1 \mathrm{mg} / \mathrm{mL}$ concentration was $143.0 \mathrm{~nm}$ (polydispersity index, 0.243). The average volume diameter of (CSO-PEI/siRNA)
HA was 149.1 (polydispersity index, 0.233). At this ratio, there was no obvious difference observed after the HA was loaded into nanoparticles. In particular, the (CSO-PEI/ siRNA)HA polyplex at the N/P ratio of 4 was $149.1 \mathrm{~nm}$ in diameter, which was optimized and used for the transfection experiments. The ability of the cationic polymer to condense RNA molecules in the nanoparticles is a necessary prerequisite for effective nucleic acid transfection of cells. ${ }^{22}$

AFM, obtained by photon correlation spectroscopy, was closer to the actual result observed than by scanning electron microscopy. ${ }^{23}$ The AFM image of (CSO-PEI/siRNA)HA is shown in Figure 3B. The nanoparticle size from AFM images was consistent with that obtained using dynamic light scattering. We observed the elliptical, spherical, and ball shape of (CSO-PEI/siRNA)HA complexes, which indicated that the siRNA molecules were compacted by CSO-PEI to form complex nanoparticles.

\section{Cellular uptake}

Using a fluorescently labeled CSO-PEI, the cellular uptake of (CSO-PEI/siRNA)HA and CSO-PEI/siRNA polyplex was estimated in IK cells by fluorescence measurement. Figure 4 shows fluorescent microscopic images after the FITC-labeled polyplex was incubated with IK cells for 1, 2, and 8 hours. The fluorescence intensity was enhanced significantly for longer incubation time. Obvious fluorescence could be

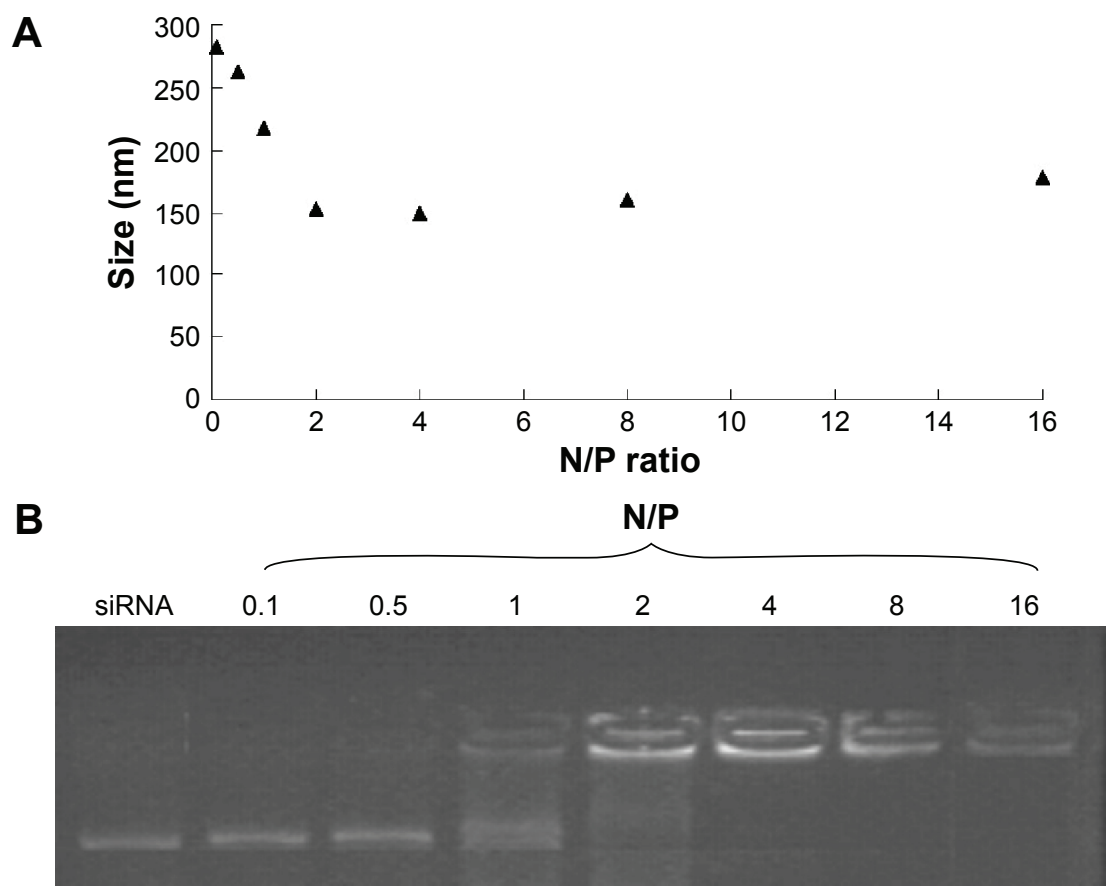

Figure 2 Particle size and gel retardation assay.

Notes: (A) Particle size and (B) gel retardation assay of (CSO-PEl/siRNA)HA polyplexes prepared at various N/P ratios from 0.1 to I6. Abbreviation: (CSO-PEl/siRNA)HA, polyethylenimine-grafted chitosan oligosaccharide with hyaluronic acid and small interfering RNA. 
A
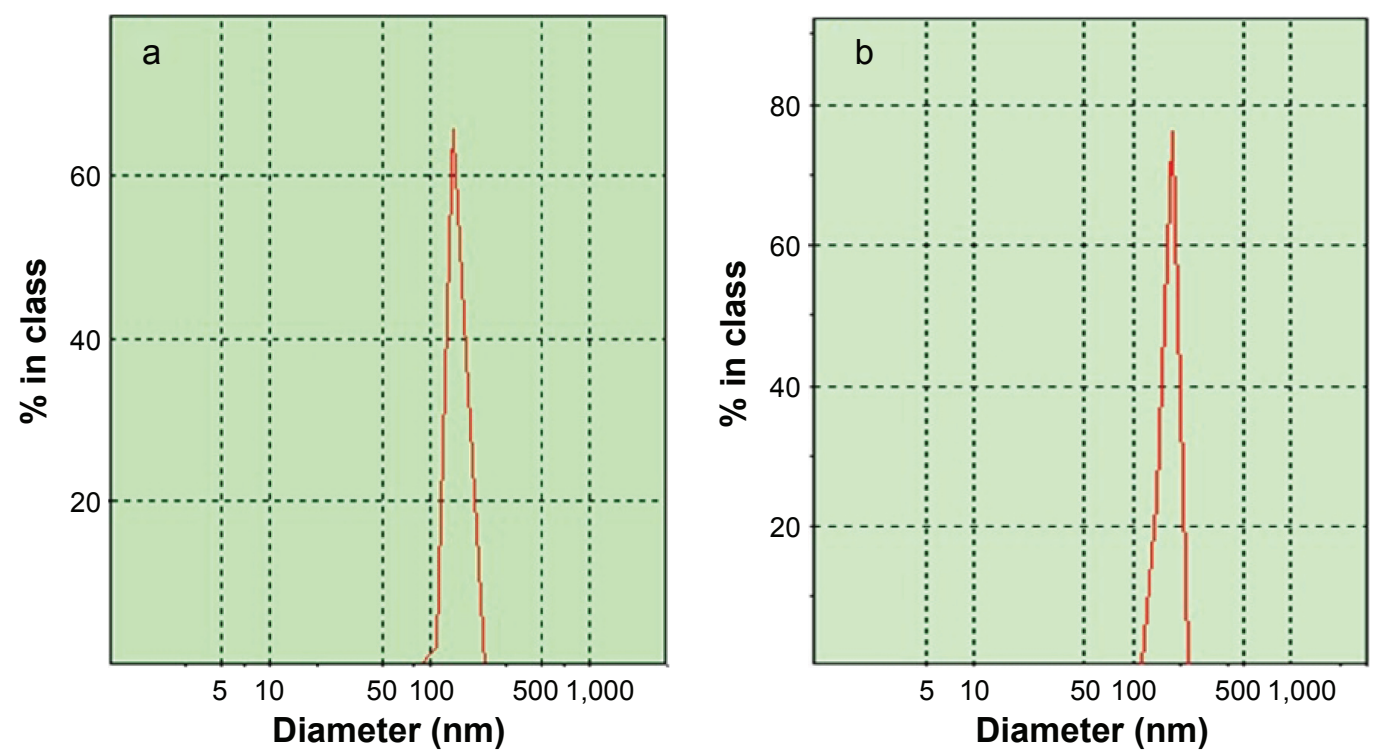

B
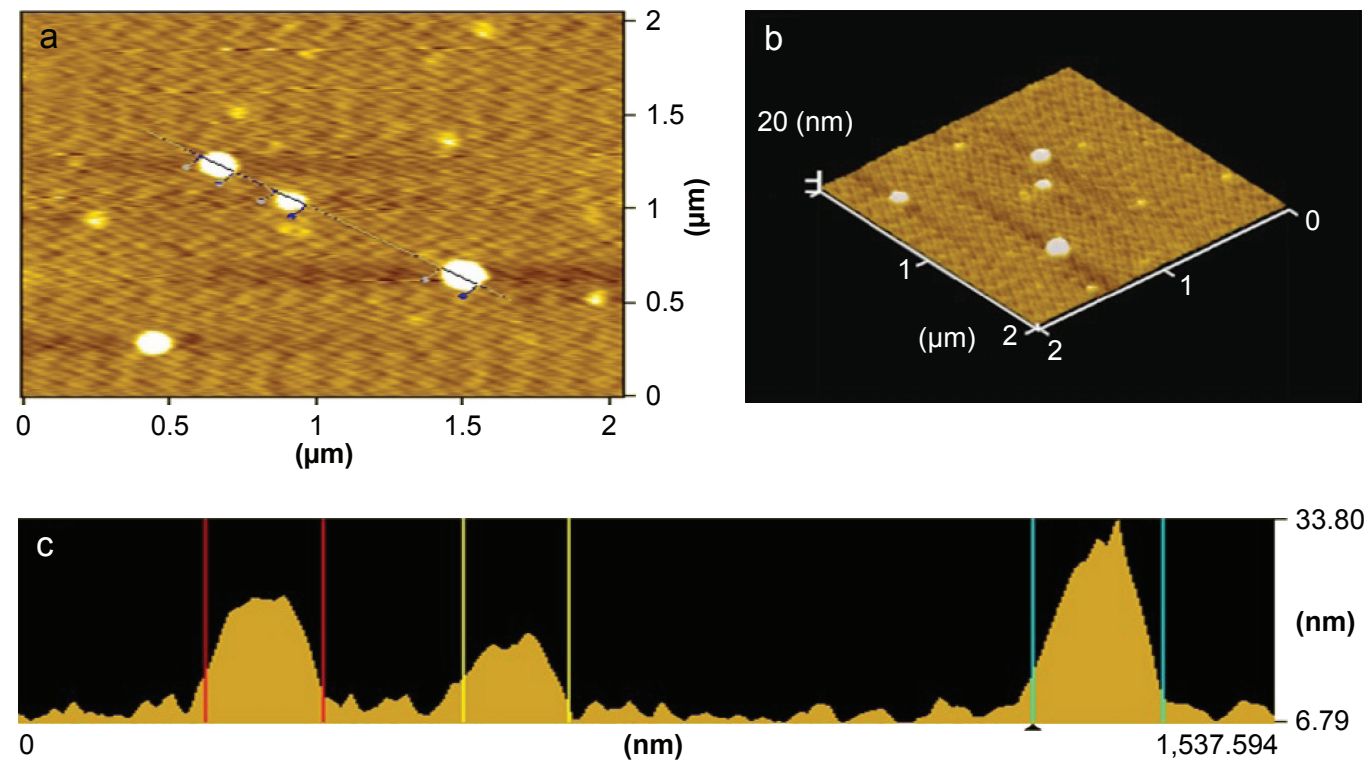

\begin{tabular}{|c|c|c|c|l|l|}
\hline & $Z 1(\mathrm{~nm})$ & $Z 2(\mathrm{~nm})$ & $\Delta Z(\mathrm{~nm})$ & Distance $(\mathrm{nm})$ & $\Phi\left(^{\circ}\right)$ \\
\hline & 13.70107 & 11.06246 & 2.638610 & 144.4316 & 1.046616 \\
\hline & 13.28088 & 9.461771 & 3.819114 & 129.3866 & 1.690713 \\
\hline & 13.71132 & 10.68695 & 3.024368 & 159.4765 & 1.086447 \\
\hline
\end{tabular}

Figure 3 The size distribution of the nanoparticles and the AFM images of (CSO-PEI/siRNA)HA.

Notes: (A) The size distribution of CSO-PEI/siRNA and (CSO-PEI/siRNA)HA: (a) CSO-PEI/siRNA and (b) (CSO-PEI/siRNA)HA and (B) AFM images of (CSO-PEI/siRNA) HA: (a) ichnography of particles, (b) stereograph of particles, and (c) the analysis of particle size.

Abbreviations: (CSO-PEI/siRNA)HA, polyethylenimine-grafted chitosan oligosaccharide with hyaluronic acid and small interfering RNA; AFM, atomic force microscopy.

detected in all cells at 24 hours. However, a noteworthy feature was that fluorescence intensity of (CSO-PEI/siRNA) $\mathrm{HA}$ in IK cells was stronger than that of CSO-PEI/siRNA within 2 hours. Furthermore, the intracellular uptake of the FITC-labeled polyplex was also conducted in a quantitative way. The average fluorescence intensity of (CSO-PEI/ siRNA)HA measured by flow cytometry after incubation for 2 hours was 12.21, which was higher than that of CSO-PEI/ siRNA (9.32). This difference decreased with the increasing incubation time. 

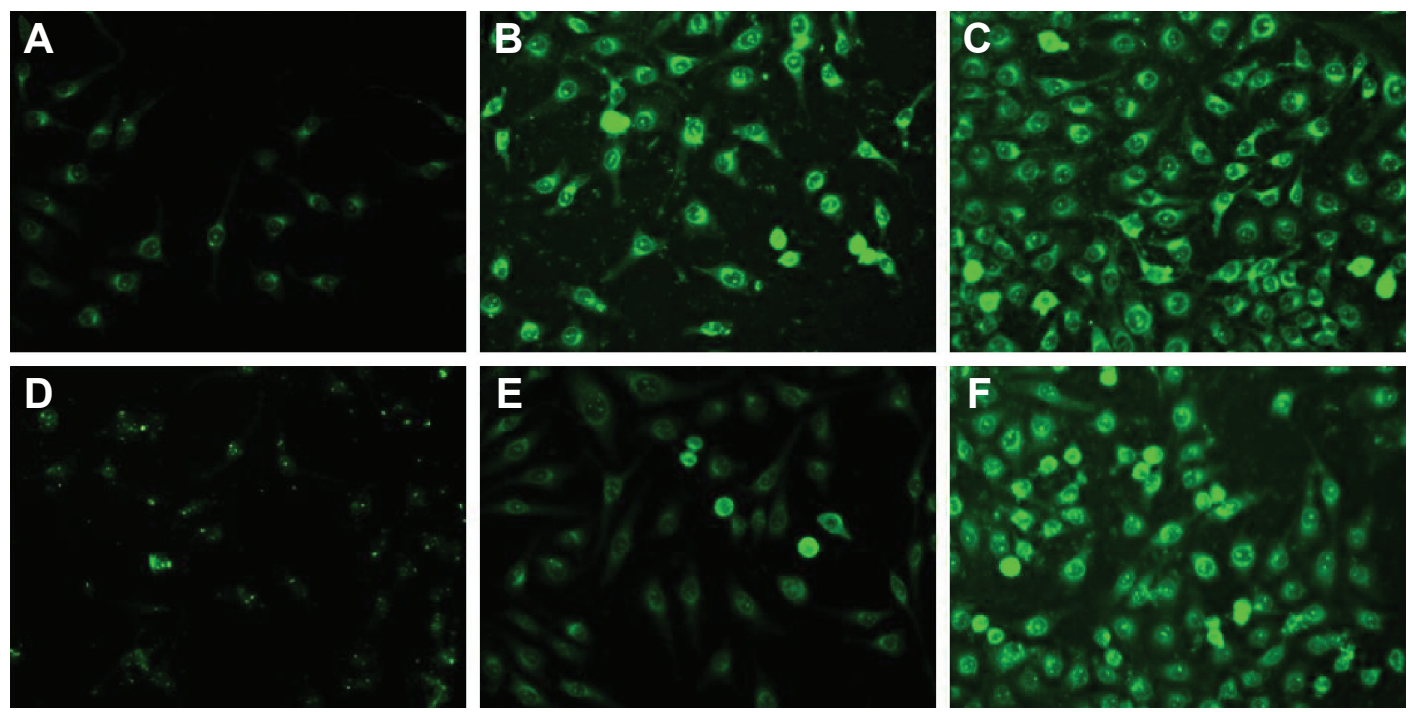

Figure 4 Fluorescence image observations.

Notes: Fluorescence image observations of the FITC-labeled (CSO-PEl/siRNA)HA incubated with the IK cells for I (A), 2 (B), and 8 hours (C) and CSO-PEI/siRNA incubated with the IK cells for I (D), 2 (E), and 8 hours (F). $\times 200$ magnification.

Abbreviations: FITC, fluorescein isothiocyanate; (CSO-PEI/siRNA)HA, polyethylenimine-grafted chitosan oligosaccharide with hyaluronic acid and small interfering RNA; IK, Ishikawa.

\section{Cytotoxicity assay of (CSO-PEI)HA}

The cytotoxicity of the (CSO-PEI)HA was investigated by MTT assay. The cytotoxicity of (CSO-PEI)HA was performed using IK cells. As shown in Figure 5, (CSOPEI)HA showed good cell viability in the cell line, and PEI was considered to have very high cytotoxicity. Furthermore, (CSO-PEI)HA had cell viability $(>50 \%$ ) at the concentration of $600 \mu \mathrm{g} / \mathrm{mL}$, which suggested that a wider range of gene transfer can be selected. Our results demonstrated that the direct modification of HA on the CSO-PEI not only shields
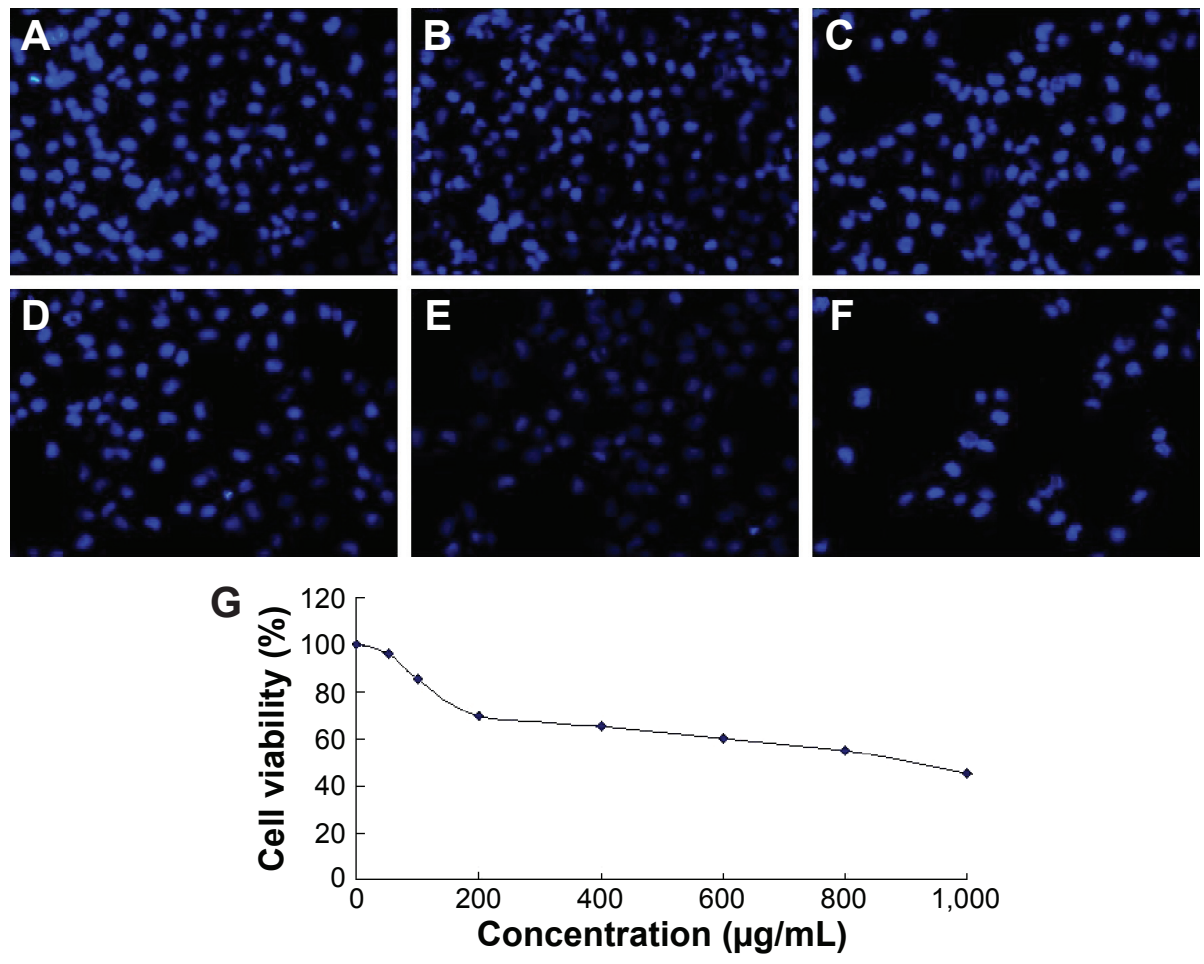

Figure 5 Cytotoxicity of copolymer.

Notes: Nuclear staining photographs were shown at concentrations of 100 (A), 200 (B), 400 (C), 600 (D), 800 (E), and I,000 $\mu$ g/mL (F), which reflects the cytotoxicity $(n=3$, error bars represent SD). Cytotoxicity of copolymer at various concentrations in IK cell lines (G).

Abbreviation: IK, Ishikawa. 
the positive charge of complex without influencing the siRNA binding but also decreases the toxicity and enhances the blood stability of the complex, similar to results from other laboratories. ${ }^{24}$

\section{Distribution in animal model}

The rat endometriosis model was thought to be successful because the endometrial explants developed into ovoid, large, fluid-filled, well-vascularized, and cystic lesions as shown in Figure 6A. To track the location of the nanoparticles, near-infrared-emitting DiR was loaded into the (CSO-PEI/ siRNA)HA and CSO-PEI/siRNA. Biodistribution and endometriotic lesion selective accumulation were analyzed by in vivo imaging after IV injection of the nanoparticle. It was obvious that fluorescence accumulation of the (CSO-PEI/ siRNA)HA in the endometriotic lesion was more significant
A
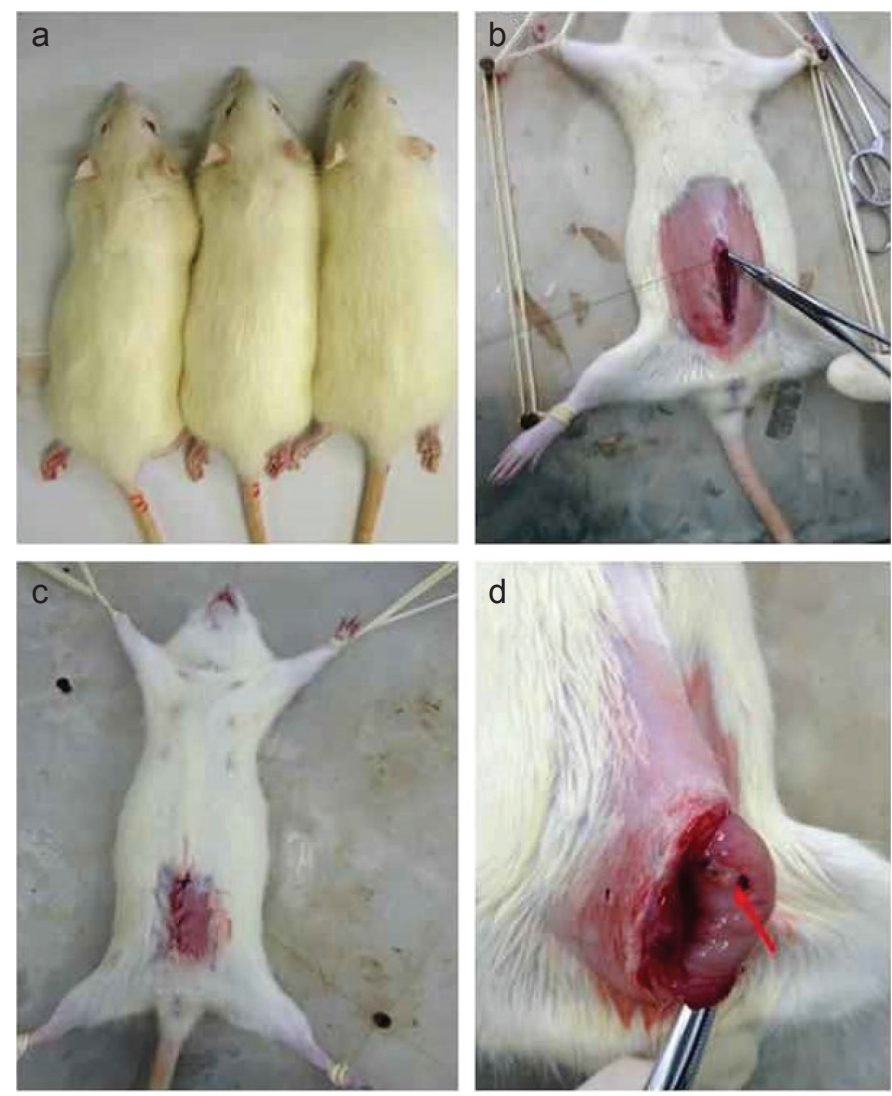

\section{B}

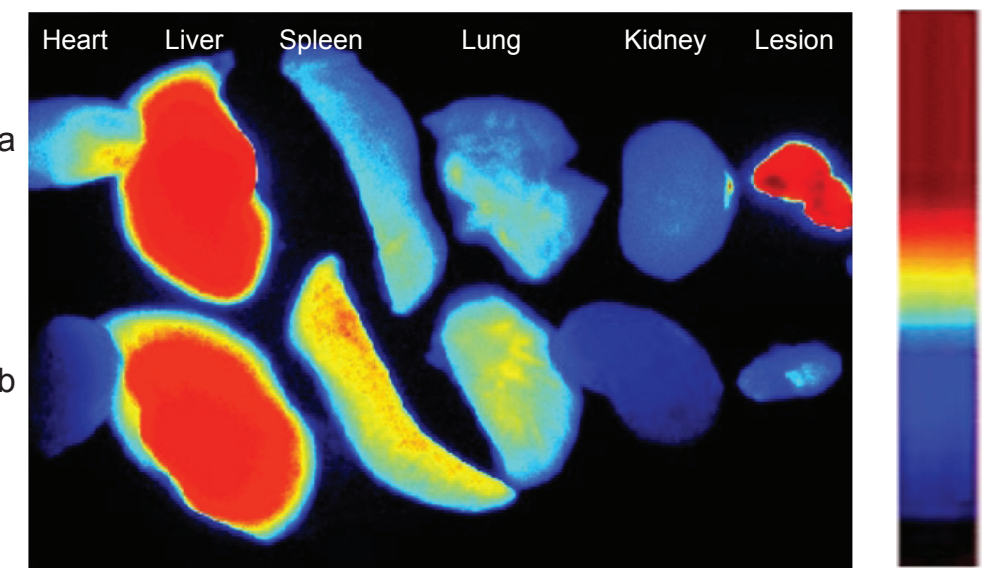

Figure 6 Procedure of making endometriosis model in rats and the distribution of fluorescent in various tissues.

Notes: (A) The figures (a-d) show the procedure of making endometriosis model in rats. It shows typical endometriotic foci, which is an ovoid, fluid-filled cystic lesion (red arrow). (B) The in vivo imaging of distribution in animal model after (CSO-PEI/siRNA)HA (a) and CSO-PEI/siRNA (b) encapsulating DIR probe injected IV, fluorescent image of various tissues ex vivo 48 hours postinjection.

Abbreviations: (CSO-PEI/siRNA)HA, polyethylenimine-grafted chitosan oligosaccharide with hyaluronic acid and small interfering RNA; DIR, I,I'-dioctadecyl-3,3,3',3'tetramethylindotricarbocyanine iodide; IV, intravenously. 
than that of CSO-PEI/siRNA during the whole experimental process (Figure 6B).

\section{Therapeutic effects}

When the implantation occurred, the average lesion volumes of the endometriotic foci were $43.88 \pm 18.85 \mathrm{~mm}^{3}$ in the danazol group, $40.39 \pm 17.19 \mathrm{~mm}^{3}$ in the control group, and $41.76 \pm 17.87 \mathrm{~mm}^{3}$ in the (CSO-PEI/siRNA)HA group. There was no significant difference among the three groups $(P>0.05)$. The sizes of the transplants in rats was treated by test and control groups and were reassessed after 2 weeks. Compared with the control group, the sizes of the transplants in rats was significantly lower for the danazol or (CSO-PEI/ siRNA)HA groups. The inhibition rates of cyst growth (\%) in the danazol and (CSO-PEI/siRNA)HA groups were $69.88 \%$ and $55.63 \%$, respectively. The lesion volumes and the sizes of the endometriotic foci were significantly decreased compared with control group.

\section{Microscopy for observation of endometriotic lesions}

Hematoxylin and eosin (H\&E) stained sections of the endometriotic lesions were examined using an optical microscope, as shown in Figure 6A. In the control group, the histopathologic findings demonstrated typical endometriotic components such as stroma and glands, and abounding hyperplasia of pseudostratified columnar epithelial cells. In the (CSO-PEI/siRNA)HA group, the structure of gland and stroma decreased obviously. We observed the gland disorganization, partly glandular atrophy, surface epithelium of cubic epithelium, or simple squamous epithelium in the (CSO-PEI/ siRNA)HA group (Figure 7A), suggesting that (CSO-PEI/ siRNA)HA might suppress the proliferation of the ectopic endometrium, leading to ectopic endometrial degeneration and atrophy. The results also suggested that AQP2-siRNA could treat the endometriosis as gene therapy.

\section{Study of adverse reaction}

The ovary and the intact uterus of the rats were weighed, and there was no significant difference between the treatment and the control groups. Reproductive organ exponent was asked for analyzing toxicity of (CSO-PEI/siRNA)HA on reproductive organs. There was no significant difference between the (CSO-PEI/siRNA)HA and the control groups, indicating that the reproductive organs were not influenced obviously by (CSO-PEI/siRNA)HA.

In addition, the reproductive organs were examined by H\&E staining with the light microscopy to determine whether the (CSO-PEI/siRNA)HA affects the structure of the ovary and uterus. The images of H\&E staining in Figure 7B show that there was no significant differences in the structures of uterus including glandular cells with matrix, which were continuous and regular, between the (CSO-PEI/siRNA)HA and the control groups. Moreover, we observed that a large amount of endometrial glandular structures was surrounded by fertile blood vessels.

Histological structures of ovaries in the control group showed the surface of monolayer cube epithelia or flat epithelium (Figure 7B). The parenchyma of ovary was divided into central medulla and peripheral cortex. Granulosa cells could be observed in the ovary cortex, and there was no significant differences in the plane structure between the (CSO-PEI/siRNA)HA group and control group.

We further examined the structures of reproductive organs using transmission electron microscopes (Figure 7C). The normal granular cells of ovarian ultra were well studied by the TEM in the control group. The density of the nucleus and the matrix was unvaried. Cells showed anomalous ellipse with a sizable nucleus. In the endochylema of the granular cells, there were abundant regular and tubiform organelles. There was no significant difference in structure between the control and the (CSO-PEI/siRNA)HA groups.

The (CSO-PEI/siRNA)HA group exhibited the same vascular density, abundant villi structure, and mitochondria, ribosome, and endoplasmic reticulum with a well-preserved morphology, which were not significantly different from the control group. Also, the nuclear membrane and intact basal membrane could be observed. Furthermore, the (CSO-PEI/ siRNA)HA group showed no mark of cell apoptosis, for example, nuclear lysis, chromatin margination at nuclear periphery, and so on. These microscopic view results were matched with the macroscopic view. The data suggested that (CSO-PEI/siRNA)HA had no adverse effects on the normal tissue.

\section{The endometrial expression of CD44}

There was no difference between the eutopic endometrial epithelial cells from the rat endometriosis model and those from the normal control rats. The epithelial cells of ectopic endometrium from rat endometriosis models showed significantly increased CD44 expression levels compared with the eutopic endometrium from the same endometriosis model. The abnormal expression of CD44 in ectopic endometria suggests that CD44 might play a meaningful role in the pathogenesis of endometriosis, as described earlier. ${ }^{18,25,26}$ 

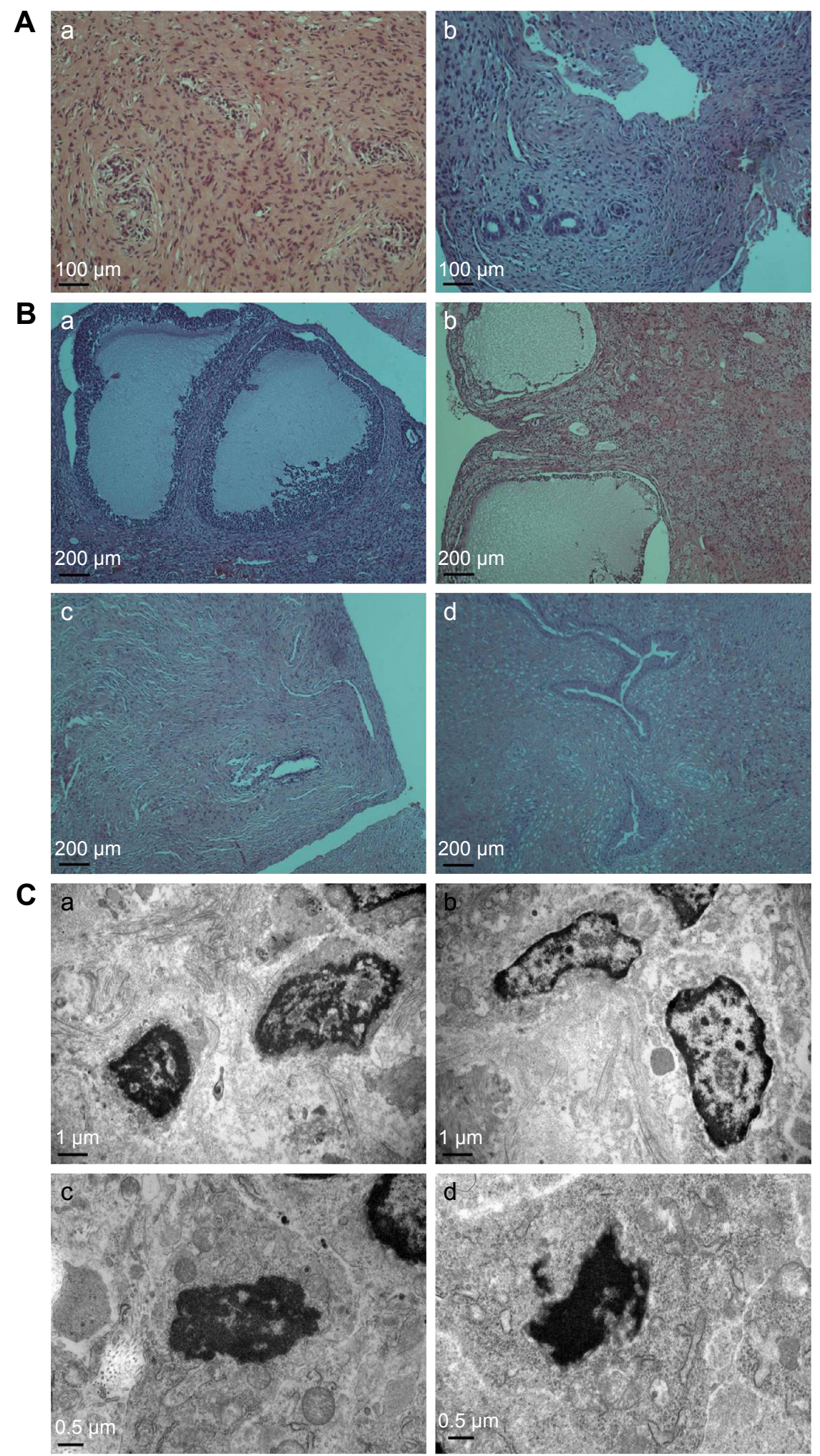

Figure 7 The light micrographs and electron micrograph.

Notes: (A) Light micrographs of endometrial lesions. In the control group, it shows abundant glands, and rich pseudostratified columnar epithelial cells can be seen clearly (a). The amount of gland structure in (CSO-PEl/siRNA)HA-treated group is less than that of the control group, and only simple squamous epithelial cells and stromal fibrosis can be observed (b). (B) The reproductive organs were examined by H\&E staining under light microscopy. The structure of the ovary of control group (a) and (CSO-PEI/ siRNA)HA group (b); the structure of the uterus of control group (c) and (CSO-PEl/siRNA)HA group (d). (C) Electron micrograph of uterus and ovary. Well-preserved morphology and intact nucleus, abundant villi, and microvillus structure of the uterus is observed in the control group (a) and (CSO-PEl/siRNA)HA group (b). The normal nuclear morphology can be seen in the control group (c) and (CSO-PEl/siRNA)HA group (d), apoptotic cells containing nuclear fragments were not found. There is no significant difference in ultrastructure between the control group and the (CSO-PEI/siRNA)HA group.

Abbreviations: (CSO-PEl/siRNA)HA, polyethylenimine-grafted chitosan oligosaccharide with hyaluronic acid and small interfering RNA; H\&E, hematoxylin and eosin. 

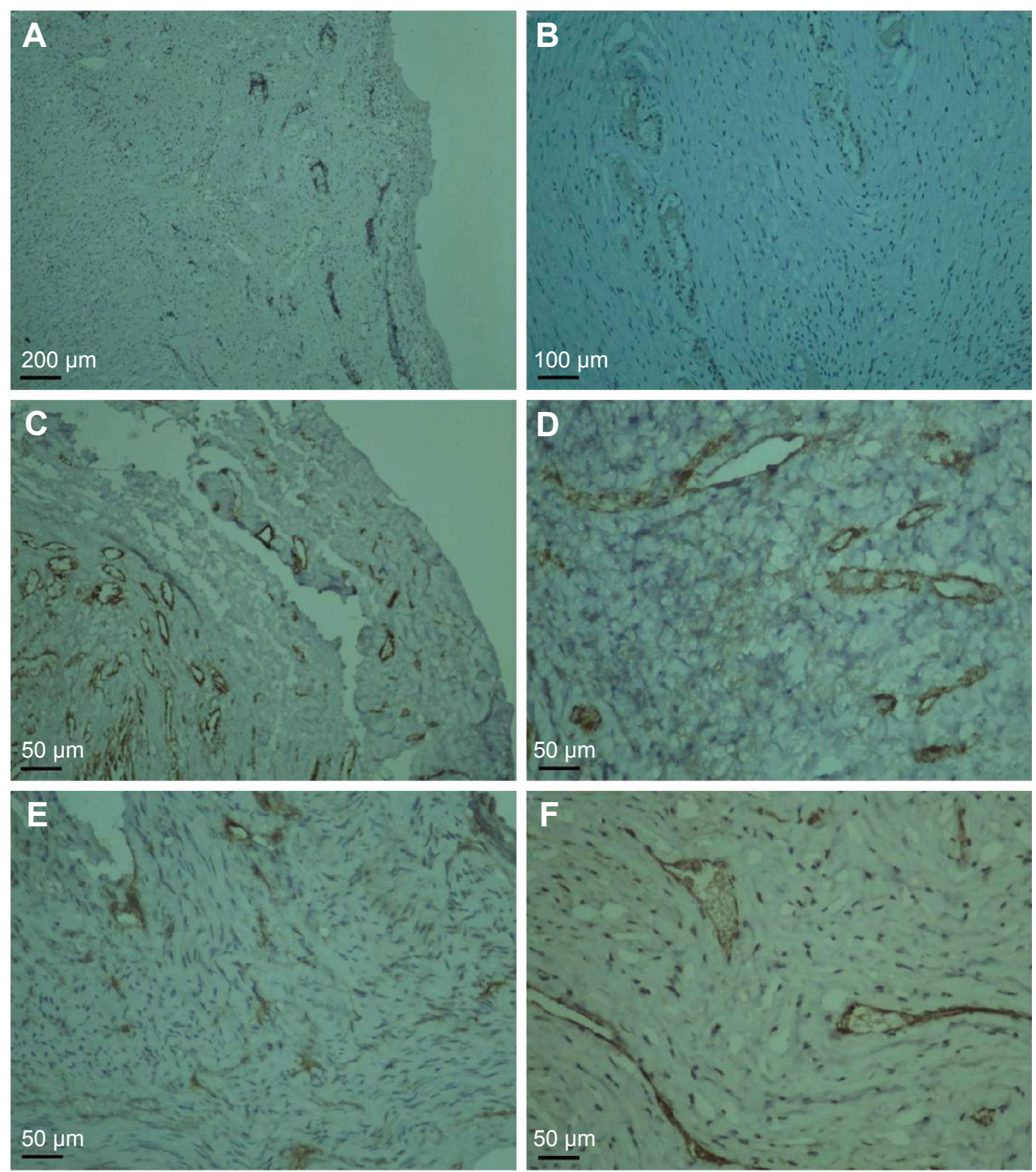

Figure 8 The expression of CD44 and MVD and cell apoptosis of endometriotic lesions.

Notes: The expression of CD44 in rat model with endometriosis in control (A) and in treatment with (CSO-PEI/siRNA)HA (B). MVD and cell apoptosis of endometrial lesions. vWF-stained MVD in control group (C) is higher than that in (CSO-PEI/siRNA)HA group (D). $\alpha$-SMA-labeled MVD showed no difference in control group (E) and (CSO-PEl/siRNA)HA group (F).

Abbreviations: (CSO-PEI/siRNA)HA, polyethylenimine-grafted chitosan oligosaccharide with hyaluronic acid and small interfering RNA; MVD, microvessel density; vWF, von Willebrand factor; $\alpha$-SMA, $\alpha$-smooth muscle actin.

From Figure 8A and B, the epithelial cells of ectopic endometrium from rat endometriosis models showed a significantly lower CD44 expression level (1.43 \pm 0.56$)$ than control (8.00 \pm 1.73$)$ after treatment with (CSO-PEI/siRNA)HA $(P<0.05$, Figure $8 \mathrm{~A}$ and $\mathrm{B})$. We also observed that the HA and CD44 could specifically bind together. ${ }^{27,28}$ The results suggest that the mechanism underlying the targeted therapy by siRNA might involve the reduced expression of CD44 in the ectopic endometria.

vWF-positive and $\alpha$-SMA-positive vessels were dispersed throughout the stroma of endometriotic lesions and focused around the glands in the control and (CSO-PEI/
siRNA)HA groups. The MVD labeled with vWF in the (CSO-PEI/siRNA)HA group was $8.19 \pm 2.35$, significantly lower than that $(25.13 \pm 3.94)$ in control group $(P<0.05$, Figure $8 \mathrm{C}$ and D). However, the MVD stained by $\alpha$-SMA in (CSO-PEI/siRNA)HA group was $8.26 \pm 0.43$, similar to that $(8.23 \pm 1.06)$ in the control group $(P>0.05$, Figure $8 \mathrm{E}$ and F). In summary, (CSO-PEI/siRNA)HA did not affect the reproductive organ exponents.

\section{Discussion}

We think that fluorescence intensity of (CSO-PEI)HA in the IK cells being stronger than that of CSO-PEI may be due to 
the following two reasons. First, (CSO-PEI/siRNA)HA and CSO-PEI/siRNA could get into IK cells with the extension of incubation time. It was attributable to the components and the special spatial structures of (CSO-PEI)HA or CSOPEI leading to excellent internalization. Chitosan modified by PEI was able to bind and protect the gene drug (siRNA) from degradation and deliver the target gene to the target cells. ${ }^{29}$ It can also prove to be a potential vector for stem cell gene therapy. ${ }^{30}$ Second, (CSO-PEI)HA could target CD44 receptors overexpressed in various cancer because the HA can specifically bind to CD44. ${ }^{31,32}$ A previous study had also reported that internalization of antibody-targeted nanoparticles was extremely dependent on the surface concentration of the ligand. ${ }^{33}$

We observed that the (CSO-PEI/siRNA)HA had better selectivity to endometriotic lesion than CSO-PEI/siRNA. Because it has been shown that HA could specifically bind to the $\mathrm{CD} 44,{ }^{34,35}$ and there is a relatively higher expression of CD44 in an endometriotic lesion, ${ }^{36}$ the (CSO-PEI/ siRNA)HA targeting distribution in vivo was more prominent than the (CSO-PEI)/siRNA. This result was consistent with the combinations between the ligand and gene vector could enhance gene transfection of the gene delivery system. ${ }^{37}$ This therapeutic effect suggested that siRNA might be carried into the cells in cysts and afterward accumulated to the lesion via the encapsulation of (CSO-PEI)HA. It is the (CSO-PEI)HA that facilitate the siRNA to deliver into the target sites. In contrast, (CSO-PEI)HA might mediate gene silencing by siRNA, suggesting that gene therapy by specific siRNAs for treatment of endometriosis may be possible. De Backer et $\mathrm{al}^{38}$ reported that in order to overcome the decrease in cellular dose, folate might be incorporated as a targeting ligand to incite receptor-mediated endocytosis, which greatly enhanced the potential of cellular uptake and gene silencing, realizing effective concentration of siRNA in the low nanomolar range.

As for the effect, the dose of (CSO-PEI/siRNA)HA might be a key part influencing the result. In contrast, the nanoparticle improves the holding of siRNA in the mouse organ and performs significant knockdown in the target gene expression for at least 10 days using a single dose of intravenous injection. ${ }^{39}$ In this study, only one dose of (CSO-PEI/ siRNA)HA was used, and more doses and plurality of time points need to be studied in the next study.

Hormonal drugs are relatively effective in the treatment of endometriosis. However, hormone therapy causes many known side effects. New drugs for endometriosis therapy with reduced side effects and increased effectiveness need to be explored. Our previous study demonstrated that AQP2 was involved in estrogen-mediated diseases, including endometriosis and endometrial adenocarcinoma. ${ }^{8}$ Decreasing the expression levels of water channel AQP2 may be a good option to treat endometriosis. In this study, we found that AQP2-siRNA was valid in the treatment of endometriosis. In contrast, accumulating evidence shows that the normal expression levels of AQP2 play important physiological roles in endometrium development, ${ }^{40}$ and decreased AQP2 expression in human endometrium was associated with reduced embryo implantation rate. ${ }^{41}$ These results indicate that decrease in the endometrial AQP2 expression may inhibit the ectopic endometrial development, which could be used as a method to treat endometriosis.

\section{Acknowledgments}

This work is supported by the National Basic Research Program of China (No 2012CB944900), the National Nature Science Foundation of China (Nos 81200428, 81173000, 81402862, 81302833, and 81300464), and the Nature Science Foundation of Zhejiang province (Nos Y2090336, z207489, and LY14H300005).

\section{Disclosure}

The authors report no conflicts of interest in this work.

\section{References}

1. Benagiano G, Brosens I, Habiba M. Structural and molecular features of the endomyometrium in endometriosis and adenomyosis. Hum Reprod Update. 2014;20:386-402.

2. Hasegawa A, Yoshino O, Osuga Y, et al. Hyaluronic acid reagent suppressed endometriotic lesion formation in a mouse model. Fertil Steril. 2010;93:2757-2759.

3. Nothnick WB. Endometriosis: in search of optimal treatment. Minerva Ginecol. 2010;62:17-31.

4. Lebovic DI, Mueller MD, Taylor RN. Immunobiology of endometriosis. Fertil Steril. 2001;75:1-10.

5. Ito $\mathrm{H}$, Shiwaku $\mathrm{H}$, Yoshida $\mathrm{C}$, et al. In utero gene therapy rescues microcephaly caused by Pqbp1-hypofunction in neural stem progenitor cells. Mol Psychiatry. 2015;20:459-471.

6. Knepper MA, Kwon TH, Nielsen S. Molecular physiology of water balance. N Engl J Med. 2015;372:1349-1358.

7. Sebastian RS, Chau E, Fillmore P, et al. Epidermal aquaporin-3 is increased in the cutaneous burn wound. Burns. 2015;41:843-847.

8. Zou LB, Zhang RJ, Tan YJ, et al. Identification of estrogen response element in the aquaporin-2 gene that mediates estrogen-induced cell migration and invasion in human endometrial carcinoma. J Clin Endocrinol Metab. 2011;96:E1399-E1408.

9. Hannon GJ, Rossi JJ. Unlocking the potential of the human genome with RNA interference. Nature. 2004;431:371-378.

10. Yan J, Du YZ, Chen FY, You J, Yuan H, Hu FQ. Effect of proteins with different isoelectric points on the gene transfection efficiency mediated by stearic acid grafted chitosan oligosaccharide micelles. Mol Pharm. 2013;10:2568-2577.

11. Hu FQ, Chen WW, Zhao MD, Yuan H, Du YZ. Effective antitumor gene therapy delivered by polyethylenimine-conjugated stearic acid-g-chitosan oligosaccharide micelles. Gene Ther. 2013;20:597-606. 
12. Zhao MD, Sun YM, Fu GF, et al. Gene therapy of endometriosis introduced by polymeric micelles with glycolipid-like structure. Biomaterials. 2012;33:634-643.

13. Lee WS, Kim YK, Zhang Q, et al. Polyxylitol-based gene carrier improves the efficiency of gene transfer through enhanced endosomal osmolysis. Nanomedicine. 2014;10:525-534.

14. Klein PM, Muller K, Gutmann C, et al. Twin disulfides as opportunity for improving stability and transfection efficiency of oligoaminoethane polyplexes. J Control Release. 2015;205:109-119.

15. Lee YM, Lee D, Kim J, Park H, Kim WJ. RPM peptide conjugated bioreducible polyethylenimine targeting invasive colon cancer. J Control Release. 2015;205:172-180.

16. Xu P, Quick GK, Yeo Y. Gene delivery through the use of a hyaluronateassociated intracellularly degradable crosslinked polyethyleneimine. Biomaterials. 2009;30:5834-5843.

17. Yao J, Fan Y, Du R, et al. Amphoteric hyaluronic acid derivative for targeting gene delivery. Biomaterials. 2010;31:9357-9365.

18. Iwase A, Kotani T, Goto M, et al. Possible involvement of CD10 in the development of endometriosis due to its inhibitory effects on CD44dependent cell adhesion. Reprod Sci. 2014;21:82-88.

19. Park J, Singha K, Son S, et al. A review of RGD-functionalized nonviral gene delivery vectors for cancer therapy. Cancer Gene Ther. 2012;19: 741-748.

20. Vernon MW, Wilson EA. Studies on the surgical induction of endometriosis in the rat. Fertil Steril. 1985;44:684-694.

21. Nap AW, Griffioen AW, Dunselman GA, et al. Antiangiogenesis therapy for endometriosis. J Clin Endocrinol Metab. 2004;89:1089-1095.

22. Dehshahri A, Sadeghpour H. Surface decorations of poly(amidoamine) dendrimer by various pendant moieties for improved delivery of nucleic acid materials. Colloids Surf B Biointerfaces. 2015;132:85-102.

23. Dubes A, Parrot-Lopez H, Abdelwahed W, et al. Scanning electron microscopy and atomic force microscopy imaging of solid lipid nanoparticles derived from amphiphilic cyclodextrins. Eur J Pharm Biopharm. 2003;55:279-282.

24. Sun Q, Kang Z, Xue L, et al. A collaborative assembly strategy for tumortargeted siRNA delivery. J Am Chem Soc. 2015;137:6000-6010.

25. Bürkle B, Notscheid KN, Scheich J, et al. Spread of endometriosis to pelvic sentinel lymph nodes: gene expression analysis. Eur J Obstet Gynecol Reprod Biol. 2013;169:370-375.

26. Heidari-Keshel S, Rezaei-Tavirani M, Ai J, et al. Tissue-specific somatic stem-cell isolation and characterization from human endometriosis. Key roles in the initiation of endometrial proliferative disorders. Minerva Med. 2015;106:95-108.

27. Kellett-Clarke H, Stegmann M, Barclay AN, Metcalfe C. CD44 binding to hyaluronic acid is redox regulated by a labile disulfide bond in the hyaluronic acid binding site. PLoS One. 2015;10:e0138137.

28. Leite Nascimento T, Hillaireau H, Noiray M, et al. Supramolecular organization and siRNA binding of hyaluronic acid-coated lipoplexes for targeted delivery to CD44 receptor. Langmuir. Epub 2015 Sep 16.
29. Kievit FM, Stephen ZR, Wang K, et al. Nanoparticle mediated silencing of DNA repair sensitizes pediatric brain tumor cells to gammairradiation. Mol Oncol. 2015;9:1071-1080.

30. Yue J, Wu J, Liu D, Zhao X, Lu WW. BMP2 gene delivery to bone mesenchymal stem cell by chitosan-g-PEI nonviral vector. Nanoscale Res Lett. 2015;10:203-213.

31. Yang X, Iyer AK, Singh A, et al. Cluster of differentiation 44 targeted hyaluronic acid based nanoparticles for MDR1 siRNA delivery to overcome drug resistance in ovarian cancer. Pharm Res. 2015;32: 2097-2109.

32. Lee GY, Kim JH, Choi KY, et al. Hyaluronic acid nanoparticles for active targeting atherosclerosis. Biomaterials. 2015;53:341-348.

33. Ragelle H, Colombo S, Pourcelle V, et al. Intracellular siRNA delivery dynamics of integrin-targeted, PEGylated chitosan-poly(ethylene imine) hybrid nanoparticles: a mechanistic insight. J Control Release. 2015;211:1-9.

34. Ebbesen MF, Olesen MT, Gjelstrup MC, et al. Tunable CD44-specific cellular retargeting with hyaluronic acid nanoshells. Pharm Res. 2015; 32:1462-1474.

35. Lee MS, Lee JE, Byun E, et al. Target-specific delivery of siRNA by stabilized calcium phosphate nanoparticles using dopa-hyaluronic acid conjugate. J Control Release. 2014;192:122-130.

36. Kim HO, Yang KM, Kang IS, et al. Expression of CD44s, vascular endothelial growth factor, matrix metalloproteinase-2 and Ki-67 in peritoneal, rectovaginal and ovarian endometriosis. J Reprod Med. 2007;52: 207-213.

37. Beloor J, Ramakrishna S, Nam K, et al. Effective gene delivery into human stem cells with a cell-targeting peptide-modified bioreducible polymer. Small. 2015;11:2069-2079.

38. De Backer L, Braeckmans K, Stuart MC, Demeester J, De Smedt SC, Raemdonck K. Bio-inspired pulmonary surfactant-modified nanogels: a promising siRNA delivery system. J Control Release. 2015;206: 177-186.

39. McLendon JM, Joshi SR, Sparks J, et al. Lipid nanoparticle delivery of a microRNA-145 inhibitor improves experimental pulmonary hypertension. J Control Release. 2015;210:67-75.

40. Zhang D, Xu G, Zhang R, et al. Decreased expression of aquaporin 2 is associated with impaired endometrial receptivity in controlled ovarian stimulation. Reprod Fertil Dev. Epub 2014 Sep 19. doi:10.1071/ RD13397.

41. Zhang RJ, Zou LB, Zhang D, et al. Functional expression of largeconductance calcium-activated potassium channels in human endometrium: a novel mechanism involved in endometrial receptivity and embryo implantation. J Clin Endocrinol Metab. 2012;97:543-553.
International Journal of Nanomedicine

\section{Publish your work in this journal}

The International Journal of Nanomedicine is an international, peerreviewed journal focusing on the application of nanotechnology in diagnostics, therapeutics, and drug delivery systems throughout the biomedical field. This journal is indexed on PubMed Central, MedLine, CAS, SciSearch $®$, Current Contents ${ }^{\circledR} /$ Clinical Medicine,
Dovepress

Journal Citation Reports/Science Edition, EMBase, Scopus and the Elsevier Bibliographic databases. The manuscript management system is completely online and includes a very quick and fair peer-review system, which is all easy to use. Visit http://www.dovepress.com/ testimonials.php to read real quotes from published authors. 